\title{
Research on Fuzzy Mathematics Model of Teaching Quality Evaluation
}

\author{
Wenting Ma \\ Nanchang Institute of Science \& Technology, Nanchang City, Jiangxi \\ Province, 330108, China
}

\begin{abstract}
Teaching quality evaluation is a very important work in education and teaching. The core of evaluation is to establish the evaluation model. The commonly used evaluation model is the average comprehensive evaluation model. The evaluation idea is based on that the evaluation result can be superimposed and the evaluation factor is based on linear relationship. In this paper, fuzzy comprehensive evaluation method is used to construct a fuzzy comprehensive teaching evaluation model based on statistics, in order to provide a scientific basis for teaching evaluation.
\end{abstract}

Keywords: fuzzy mathematics model, teaching quality evaluation

\section{Introduction}

At present, all colleges and universities in our country are beginning to fully realize the importance of teaching evaluation, and gradually take teaching quality assessment as one of the daily work of teaching management. Evaluation of teaching quality for teachers in colleges and universities generally through student evaluation, expert evaluation, peer evaluation and teacher self-evaluation of the four links, and then comprehensive consideration of the final evaluation results. However, there are still some unsolved problems in the process of establishing, using and evaluating the results, such as the research of evaluation theory, the updating of evaluation methods and the analysis of evaluation data and so on. These problems all affect the realization of the teaching quality evaluation function and the teachers' potential ability excavation in varying 
degrees. In order to achieve the comprehensive index of teaching evaluation system and the balanced rationality of the weight distribution of the indexes, a more comprehensive and dynamic evaluation of a teacher's true teaching quality, the author establishes a fuzzy evaluation model of teaching process with fuzzy mathematics method.

The core of the evaluation is to establish the evaluation model. The common evaluation model is the weighted average model of the evaluation index. The core of the evaluation theory is based on that the evaluation result can be superimposed and the evaluation factor can be superimposed. The evaluation factor is the most important one. This model is simple to calculate, easy to model, most of the current teaching evaluation using this method. But in fact the prerequisites for the establishment of the model can not be fully established, so they are often not very satisfied with the application, the credibility of the evaluation results are also low. In fact, the evaluation object contains a variety of attributes, these attributes reflect the evaluation of different characteristics of objects, and these features are often with a certain degree of ambiguity, so the fuzzy mathematical approach to comprehensive evaluation, will be closer to In the actual situation, based on this idea, to build on the basis of statistics based on fuzzy comprehensive evaluation model of teaching.

\section{Theoretical Basis of Fuzzy Comprehensive Evaluation}

Fuzzy comprehensive evaluation is based on the nonlinear characteristics of the evaluation process, which is based on the fuzzy algorithm in fuzzy mathematics, the quantitative evaluation of non-linear domain synthesis, resulting in comparable quantitative evaluation results.

When the evaluation object is selected, the evaluation factors involved in the evaluation are classified and stratified scientifically and rationally. The evaluation index system with the combination of testability, completeness and feasibility is established. As the single factor, The role of the different evaluation index to set the weight of the proportion of its evaluation vector, the collection of reviews for the collection, but also to determine the value of the assessment level, that is, assess the degree of membership, to obtain quantitative comments Then, according to the evaluation index system, we design the sample collection table, carry out the sample collection and data processing, and substitute the fuzzy evaluation formula into the mathematical model. The formula, the evaluation results, and finally the results of the evaluation should be tested to determine whether the results are credible.

Evaluation is to achieve a certain purpose, the use of specific indicators, compared with a unified standard, the use of prescribed methods, to make a value judgment of things a cognitive activity, evaluation is the level of human development to a certain stage of the product, It is generally believed that evaluation has four functions, namely, judgment function, prediction function, choice function and guidance function. Among them, the guiding function is the most important, and the evaluation is the most important one. Core position, the 
first three functions is the fourth function of the foundation and process. The core of the evaluation is to establish the evaluation model, the most well-known evaluation model is linear, that is, the weighted average of evaluation indicators integrated model, the evaluation ideas based on the evaluation results can be superimposed on the assumption that the linear relationship factor, which This model is simple in calculation and convenient in modelling. At present, most of the existing teaching evaluation adopts this method, but in fact this assumption can not be established. So it is often unsatisfactory to use and the credibility of the evaluation result is poor., When the various factors are fuzzy concepts, weights are also vague, the traditional total score and weighted average and other methods of calculation does not apply, in fact, teaching evaluation object contains a variety of attributes, these attributes from different sides Reflecting the different characteristics of the evaluation object, and these features are often with a certain degree of ambiguity, that is, has a nonlinear characteristic, so the use of fuzzy mathematics method for comprehensive evaluation, will be closer to the actual situation.

\section{The construction of fuzzy comprehensive evaluation model}

The basic idea of the fuzzy comprehensive evaluation method is to construct the fuzzy judgment matrix by using the fuzzy set transformation principle and the membership degree to describe the fuzzy boundary of each factor and factor on the basis of determining the evaluation standard and the weight value of the evaluation factor and factor. Multi-layer composite operation, and ultimately determine the evaluation object belongs to level.

There are $\mathrm{n}$ evaluation grades, $\mathrm{m}$ first-level evaluation indexes (factors) and each level indicator contains k second level indicators (factor), and use U, V, Vi and other symbols, namely: evaluation of the set of categories $U=\{\mathrm{V} 1, \mathrm{~V} 2$, $\mathrm{Vm}\}$ is evaluated by evaluating the factor set $\mathrm{V}=\{\mathrm{V} 1, \mathrm{~V} 2,, \mathrm{Vm}\}$. Because there is a fuzzy relation $\mathrm{R}$ between $\mathrm{U}$ and $\mathrm{V}$, it can be expressed as a fuzzy matrix

\section{The establishment of evaluation sets}

In the actual teaching quality evaluation system, in order to make the evaluation results have a strong scientific and accuracy, the evaluators are generally composed of various types of personnel, mainly education experts, school leaders, peer teachers, students and so on. The evaluation results of different kinds of personnel are different and the calculation method is as follows: firstly, the comprehensive evaluation results of all kinds of appraisers are obtained; finally, the conclusion of fuzzy evaluation is obtained.

The evaluation results are excellent, good, medium, qualified, unqualified five grades, recorded as $\mathrm{Vc}=$ (excellent, good, medium, qualified, unqualified), as the evaluation of the concentration level is fuzzy, its rating is a certain The numerical 
value of the change in the range, inconvenience calculation comparison, not intuitive, so in practice, the evaluation of the various levels with a specific numerical representation. If the percentage system for the evaluation criteria, excellent teaching quality, results should be 90 to 100 , the median value of 95 ; good at 80 to 89 , the median value of 85 ; medium in the 70 to 79 , the median value of 75 ; teaching quality, The results in 60 to 69 , the median value of 65 ; teaching quality is not qualified, temporarily consider the results in 50 to 60 (less than 50 are not suitable for teachers), the median value of 55 . Then we get the standard set of teaching effect percentage $\mathrm{V}=(95,85,75,65,55)$.

\section{Evaluation factor set}

This model is different from the traditional simple average algorithm, but the use of fuzzy comprehensive evaluation of the method, the method is relatively common evaluation method to weigh all kinds of factors, to ensure maximum retention of information, truly participate in the assessment process of each $\mathrm{A}$ person's evaluation information can play a corresponding role, so that a comprehensive and dynamic evaluation. To realize the function goal of the evaluation model, that is to evaluate the teaching quality of teachers, we must first determine the evaluation index system. The findings of the current survey found that most institutions use quantitative assessment of teaching quality indicators system, that is, the teaching content of teachers, teaching methods, teaching attitude, teaching effectiveness and teaching ability to assess the content.

\section{Analysis of Evaluation Case}

According to the above principles, a school teacher evaluation of mathematics as follows.

\subsection{Single factor evaluation}

The number of participants is 40 and the evaluation grade is 5 , that is: excellent, good, medium, qualified, unqualified, the evaluation statistics are shown in Table 1. Among them, the degree of membership in the curriculum preparation is $12 / 40$, $18 / 40,8 / 40,2 / 40,0 / 40$ respectively. According to Table 1 , we can get the membership degree of each evaluation factor with respect to each level, and then list the fuzzy relation matrix, finally get the fuzzy evaluation result (the weight (value) in the following calculation comes from the reference.

\subsection{Multi-factor comprehensive evaluation}

The evaluation of teaching effectiveness is a very serious matter, must be based on a fair, open, fair, serious and responsible principles, a single evaluation from a single, there may be malicious score phenomenon, the final result So the actual 
evaluation can be considered by the education experts, leaders, peers, students and other components, their weight (value) in accordance with the experience to determine the judge can also be obtained in accordance with the AHP, the final determination of $0.5,0.2,0.2,0.1,0$. Using the above method can be obtained from different types of evaluation of single-factor comprehensive evaluation results.

\section{Conclusion}

Through the comprehensive analysis of the teaching quality evaluation factors, the fuzzy comprehensive evaluation model of teaching quality conforms to the situation of our country is established, which provides a strong basis for impartial and objective evaluation of teaching quality. The correct evaluation of teaching effectiveness helps teachers improve teaching methods, overcome the problems in the teaching process, it has important practical significance to improve the teaching quality and promote education reform.

\section{References}

[1] Feng Yongchao. Methods to improve the scientific nature of educational evaluation, Educational Research, 55(12), pp.68-70, 2011

[2] Tang Xianjun. Discussion on several evaluation methods of new mathematics teaching. Journal of Education, 8 (5), pp.87- 91, 2013

[3] Yang Lunbiao.Fuzzy mathematical principle and its application. Guangzhou: South China University of Technology Press, pp. 18- 20, 2012

[4] Liu Lin, Liu Zhixin, Wu Zhongqiang. Fuzzy evaluation system and practice of teaching quality in colleges and universities. Journal of Shijiazhuang University of Economics, 9(6), pp.58- 61, 2011

[5] He Zhongxiong. Fuzzy mathematics and its application. Tianjin: Tianjin Science and Technology Press, pp.85-87, 2014 